\title{
ENJEUX FAMILIAUX AUTOUR DE LA FÊTE DE NOËL
}

\author{
Évelyne Favart
}

ERES | « Dialogue »

2005/2 n $168 \mid$ pages 107 à 113

ISSN 0242-8962

ISBN 2749204283

Article disponible en ligne à l'adresse :

https://www.cairn.info/revue-dialogue-2005-2-page-107.htm

Distribution électronique Cairn.info pour ERES.

(C) ERES. Tous droits réservés pour tous pays.

La reproduction ou représentation de cet article, notamment par photocopie, n'est autorisée que dans les limites des conditions générales d'utilisation du site ou, le cas échéant, des conditions générales de la licence souscrite par votre établissement. Toute autre reproduction ou représentation, en tout ou partie, sous quelque forme et de quelque manière que ce soit, est interdite sauf accord préalable et écrit de l'éditeur, en dehors des cas prévus par la législation en vigueur en France. Il est précisé que son stockage dans une base de données est également interdit. 


\title{
Actualité des pratiques et de la recherche
}

\section{Enjeux familiaux autour de la fête de Noël}

\author{
EVELYNE FAVART
}

Quand on parle de la famille, on a tendance à réduire celle-ci à une unité de résidence. On ne peut pas non plus admettre que la famille soit seulement constituée d'un couple, dans ses formes classiques ou recomposée, et de ses enfants. La dimension collatérale et surtout intergénérationnelle des rapports familiaux doit aussi être prise en considération, d'autant que l'allongement de l'espérance de vie entraîne que trois, voire quatre générations se côtoient, sources d'échanges matériels, affectifs, sociaux et symboliques. Le rituel annuel de la fête de Noël illustre bien ces dimensions.

Aujourd'hui, la famille a changé, elle est, par certains aspects, moins inscrite dans la durée qu'auparavant, mais elle reste codée en termes positifs pour la majorité des individus contemporains. Les familles d'aujourd'hui semblent marquées par deux caractéristiques : la fragilité conjugale et la co-longévité des générations.

Dans cet article, nous étudierons les enjeux familiaux autour de la fête de Noël, à partir d'une étude qualitative constituée d'entretiens avec des individus d'âge adulte ${ }^{1}$. Quel sens les personnes interviewées donnentelles à cette fête, de quelle manière est-elle vécue ? Comment fête-t-on Noël en famille dans le contexte actuel de bouleversement des temporalités familiales, d'autant que cette célébration serait " l'emblème du home sweet home » (Perrot, 2000, p. 94) ? Bien entendu, la façon de fêter Noël donne aussi des indications sur la situation socio-économique des familles, rappelant leurs trajectoires ou parfois leurs particularismes régionaux. Toutefois, dans cet article, cet aspect ne sera pas envisagé ; il s'agit plutôt de se pencher sur la fête de Noël en tant qu'elle permettrait la préservation d'une intimité familiale, laquelle 
deviendrait l'objet sacré le temps d'une célébration.

\section{Noël, fête familiale par excellence?}

En principe, d'après les interviewés, « la » famille se réunit systématiquement pour fêter Noël, autour d'un repas festif. D'après de Singly (1993), la fête de Noël est une « mise en scène de la famille et de la parenté devant elle-même » (p. 81, op. cit.). En témoigne le flot de photos décorant les albums familiaux, qui atteint des sommets impressionnants au moment de Noël (Kaufmann, 2002).

D'après une interviewée : « Il y a beaucoup d'amour entre nous et on le montre très fort ce jour là ${ }^{2}$. » Dans ce sens, la fête de Noël permet aux membres de la famille en présence de confirmer l'attention et l'affection qu'ils se portent, en exposant aussi ces liens dans des cadeaux. Aux États-Unis, Caplow (1986) a montré que le rituel des cadeaux était « une méthode de traitement des liens importants mais fragiles, qui consiste à offrir des cadeaux à des personnes ou des collectivités dont la bienveillance est souhaitée mais ne peut être tenue pour acquise ou allant de soi » (p. 45). Lors de la fête de Noël, les uns et les autres s'échangent des marques d'attention, notamment à travers les cadeaux, censés représenter des liens relationnels, sous les yeux attentifs du cercle de famille. Cette prestation relève bien de la mise en scène, de nature familiale.

Une norme qui a caractère d'évidence autour de Noël est celle qu'il requiert de le fêter en famille, prônant le culte de l'entre-soi : « Dans ma famille, Noël, c'est avant tout une fête de famille, c'est-à-dire un moment où on aime que tout le monde soit rassemblé pour passer un bon moment ensemble ${ }^{3}$. " Le mot d'ordre serait donc celui du rassemblement familial. Le rituel est bien d'ordre familial : d'une part, il se célèbre en famille (tous les individus qui y participent, à de rares exceptions près, partagent des liens de parenté) et d'autre part, la charge symbolique est de nature familiale (la fonction principale est une fonction d'intégration du groupe familial). En outre, les échanges ont très majoritairement pour thème la famille et ses différents membres, au passé, présent et devenir. La simple coprésence des individus reflèterait cette appartenance, voire la renforcerait.

Mais quelle famille s'agit-il de rassembler? Lorsque les propos des personnes interviewées sont approfondis, on perçoit qu'il y a inévitablement sélection familiale. Ainsi, une dame ${ }^{4}$ qui, en début d'entretien, affirme fêter Noël « tous ensemble en famille », admet par la suite que des mésententes familiales ne permettent pas d'inviter les frères et sœurs de son mari, lesquels refusent catégoriquement de se rencontrer. En outre, les recompositions familiales ont des répercussions sur la participation au rituel. Ainsi, une interviewée a raconté que suite au décès de son frère, la femme et les enfants de ce dernier ont encore participé à la fête de Noël chez les parents, jusqu'à ce que la belle-sœur se soit remariée, exprimant alors le désir de fêter Noël dans sa nouvelle famille, ce qui a été très mal perçu par la famille de son premier mari. 
D'une façon générale, les équipes conjugales ne se séparent pas lors de la fête de Noël, ce qui donne un indice du primat du conjugal. Souvent, la fête est dédoublée : par exemple, la famille conjugale de procréation se rend le 24 décembre dans la famille d'orientation de l'un des deux partenaires et le 25 , dans celle de l'autre conjoint. D'après Caplow, pour les États-Unis : « Dans la plupart des cas, cette réunion de famille se compose d'une cellule parentenfant (un ou deux parents avec un ou plusieurs enfants) et de personnes qui sont rattachées à cette cellule par l'intermédiaire d'autres cellules parents-enfants (enfants des enfants, conjoints des enfants, frères ou sœurs des parents, ou parents des parents) » (Caplow, 1986, p. 57). Il semble très occasionnel de réunir ensemble les deux familles d'orientation des conjoints.

Concrètement, une famille l'emporte-t-elle sur l'autre ? Les négociations familiales autour du comment fêter Noël peuvent se passer sans heurts. Parfois, lors de recomposition familiale, les ex-conjoints peuvent trouver un arrangement afin que personne ne soit seul pour fêter Noël, ce qui semble consister une règle prédominante : "Je suis divorcée, les enfants passent toujours Noël chez leur père, car leur père n'a plus de famille. Alors pour qu'il ait quand même une famille, puisque c'est Noël, nous avons décidé que ce serait toujours comme ça. Donc le 24, je vais chez mes parents avec mes frères et sœurs, mes beaux-frères et mes belles-sœurs et les enfants de certains de mes frères et sœurs ${ }^{5}$. " Une grand-mère nous a expliqué que depuis quelques années, elle n'avait plus l'occasion de réunir ses enfants et petits-enfants la veille de Noël car ces derniers se rendaient dans leur belle-famille : «Il faut bien qu'ils partagent, ils ne peuvent pas se couper en deux ${ }^{6}$. » Le rituel a simplement été déplacé à un autre moment. Pour certains, le dédoublement de la fête semble constituer une nécessité : «Il y avait deux familles, donc on était obligé de se partager ${ }^{7} »$, alors que dans d'autres familles, une famille l'emporte sur l'autre, plaçant dans l'ombre des pans entiers d'une lignée familiale. Les bricolages familiaux autour de la fête de Noël sont donc diversifiés, mais ils respectent, tant que faire se peut, un certain nombre de règles, comme celle invitant à ne pas laisser une personne seule lors de cette occasion ou celle ne permettant pas de séparer les équipes conjugales.

\section{Noël, fête des aînés ?}

Parfois, la fête de Noël mélange gaieté et chagrin : le bonheur des retrouvailles familiales se mêle à la douleur à l'évocation d'un parent qui n'est plus. D'après une interviewée : « C'est un si beau jour... Mais pour moi, c'est aussi un jour de tristesse en même temps [...] C'est merveilleux mais d'un côté, je suis un peu triste parce que j'ai perdu ma maman il n'y a pas si longtemps et mes pensées vont vers elle, mais en même temps, c'est un beau jour ${ }^{8}$. " Noël est aussi l'occasion d'évoquer ensemble ces êtres chers disparus mais qui restent bien ancrés dans la mémoire familiale. N'est-ce pas aussi cela une famille, l'ensemble 
des personnages, vivants et morts qui restent en mémoire ?

Lorsque l'avenir est incertain, lorsqu'on ressent bien que la disparition des aînés correspond à un futur proche, la volonté de rassemblement est encore plus manifeste. « Noël nous donne toujours l'occasion de nous retrouver, surtout quand les parents vieillissent, on se dit : encore cette année, l'année prochaine peutêtre plus ${ }^{9} \ldots$.. $>$

Si les aînés de la famille occupent une place symbolique de premier ordre, leur disparition entraîne régulièrement une modification du rassemblement familial à l'occasion de la fête de Noël, car les contours de la famille se redessinent. Tant que les aînés sont en vie, l'obligation familiale prime et semble peu remise en question : on ne se permet même pas d'envisager de célébrer Noël autrement : " Jusqu'à maintenant, Maman est là, donc on ne se pose même pas la question [...] Les frères et sœurs, beaux-frères et belles-sœurs, on sait très bien qu'on doit réserver ce jourlà pour mes parents. Tant que ma mère saura faire son petit repas familial, tant qu'elle sera là, elle veut absolument continuer et que tout le monde se réunisse ${ }^{10}$. » La qualité des relations intergénérationnelles s'entremêle au sentiment du devoir, qui est perçu positivement, comme un devoir moral que l'on accomplit volontiers. Une dame, infirmière, en témoigne : « Ça ne m'a jamais pesé. Moi, ça ne me dérangerait pas de travailler le soir de Noël, mais disons que pour la famille... J'estime que je suis obligée d'être présente, enfin pas obligée, mais disons qu'il vaut mieux quand même que je sois là et c'est une bonne chose ${ }^{11}$. »

Il semble que ce soit majoritairement les aînés qui « tiennent la famille », tant qu'ils sont en vie, c'est-à-dire que le temps d'un soir, celui de Noël, c'est leur conception $\mathrm{du}$ « nous famille » qui prédomine et qui est peu remis en question. Ils peuvent parfois imposer leur propre définition des contours internes de la famille, se traduisant concrètement en associant ou non certaines personnes à ces rassemblements. Ainsi, Anne-Marie ${ }^{12}$, une interviewée, a raconté que malgré la séparation d'avec son mari, sa mère a persisté à inviter son ex-beau-fils à l'ensemble des réunions rassemblant la famille, en estimant qu'il restait le père de ses petits-enfants. Le nouveau conjoint d'Anne-Marie n'a été toléré qu'au moment de l'officialisation de leur union, soit cinq longues années plus tard, et sans que l'ex-conjoint ne cesse pour autant d'y être convié. Actuellement, Anne-Marie se rallie au positionnement de sa mère, estimant que pour ses enfants, c'était une bonne formule, mais ne cache pas que cette situation a été très embarrassante pour elle.

Parfois les aînés, par-delà leur mort, peuvent encore inciter au rassemblement : ce dernier est alors perçu comme un signe de fidélité ou d'affection commun envers un parent disparu. Toutefois, au fil du temps, Ego peut ressentir un essoufflement : "Cette année, on a fait Noël chez une de mes sœurs, mais finalement, vous savez comment ça a tourné ? On s'est retrouvé entre nous, c'est-à-dire que chacune de mes sœurs était au milieu des jeunes, 
mais avec ses propres enfants. Alors à quoi ça sert si chacun est quand même avec ses enfants ? À quoi ça sert tout ce chahut avec trente personnes ? Quel est le but alors ${ }^{13}$ ? » En somme, cette dame ne perçoit plus le sens du rassemblement, car le « nous familial » prioritaire serait celui qui relie aux familles de procréation respectives. Le rituel est censé manifester le signe du lien familial, en donnant une consistance à la famille, un point d'ancrage dans le temps présent. Dans ce cas, le rassemblement est devenu essentiellement une formalité, une coquille vide; le rituel détient essentiellement une fonction phatique.

$\mathrm{Au}$ décès des aînés, il n'est pas rare que les langues se délient et que la dynamique familiale prenne une autre tournure : «Ce qui est triste dans cette affaire, c'est une famille qui était très unie, mais il a fallu que maman meure pour que les antagonismes se révèlent ${ }^{14}$. » On le sait, le moment de la disparition des parents âgés et de l'héritage ${ }^{15}$ peut constituer une secousse familiale causant de nombreux dégâts et ayant notamment des répercussions sur le rituel annuel autour de la fête de Noël.

\section{La trêve familiale de Noël}

Avec Noël, une autre exigence consiste à ce que les familles qui se rassemblent tentent de gommer les tensions et les conflits. Explicitement, il s'agit de se réunir physiquement et de laisser de côté tout sujet de conversation qui pourrait semer la zizanie. «Au niveau de l'ambiance, en général, ça se passe très bien. On ne règle pas nos problèmes lors de cette fête-là, on essaie que tout se passe pour le mieux. En fait, on est là pour manger ensemble, pour bien boire et bien manger et passer du bon temps. On n'est pas là pour se chercher des problèmes, on est là pour être ensemble et pour s'apprécier, on est là pour fêter Noël ensemble ${ }^{16}$. । Toutefois, les non-dits peuvent planer et les incompréhensions perdurer. Des piques à peine voilées peuvent s'échanger entre les participants : « Il n'y a pas de disputes, parce que je pense que tout le monde est trop bien éduqué pour ça. Mais les piques s'échangent [...] Malgré tout, on est face à des adultes qui savent quand ils doivent se taire [...] Il n'y a pas de gros conflits mais il y a beaucoup de non-dits et on reste sur ces non-dits et peut-être que si l'abcès était crevé, la relation n'en serait que meilleure ${ }^{17} \ldots$..

Il existe ainsi des évidences par rapport à Noël, et notamment celle qui requiert que cette fête représente un hymne familial. « Familialement, c'est le temps où l'on se rapproche » confiera une des interviewées. En somme, ces réunions ne sont pas censées rencontrer prioritairement le « souci de soi »(de Singly, 1996), d'autant plus lorsque ce dernier influencerait de façon négative l'ambiance festive du rituel. Ainsi, un interviewé dénonce certaines règles propres à Noël : «Par exemple à Noël, ça se fait d'avoir une figure de fête. Ça ne se ferait pas d'arriver pas bien habillé ou fatigué ou découragé parce qu'on a un problème ${ }^{18}$. " Chacun des participants est ainsi tenu de faire la meilleure figure possible, toutefois des ratés peuvent venir se 
faufiler dans cette mécanique bien huilée.

\section{Le désordre familial de Noël}

Les tensions familiales peuvent pourtant prendre le dessus, car les relations familiales sont fragiles. Quand le conflit est déclenché, la famille entre alors dans une zone de turbulence relationnelle. Certains peuvent alors s'abstenir de participer au rituel. Ego peut choisir d'éviter d'offenser les participants, en se protégeant derrière un solide alibi. La meilleure parade consiste à partir en vacances à l'étranger, accompagné de sa famille conjugale. Lors de l'entretien, on confie les vrais motifs de l'absence, mais face à la famille, on propose une version officielle non offensante : «On essaie de maintenir une certaine tradition, le fait de se réunir à Noël, bien qu'il y ait eu des moments où c'était un peu tendu et difficile et ça m'arrangeait très bien de ne pas être là. On aime bien le ski, mais on pouvait évidemment choisir une autre semaine. Je vous le dis franchement, je ne leur ai pas dit à elles, je disais que ça s'arrangeait comme ça. Et mon mari n'avait pas non plus envie de voir ma famille ${ }^{19}$. » Cette forme d'abstention peut alors être décodée comme une barrière protectrice pour le moi intime et le moi conjugal. Ainsi, d'après une autre personne interviewée, éviter la fête familiale de Noël est « essentiel pour mon équilibre et celui de mon couple ${ }^{20} »$. Ego peut donc adopter une démarche auto-protectrice, se mettre à l'abri, en fuyant des rassemblements qui occasionnent un coût personnel considéré excessif.

Mais Ego peut aussi refuser catégoriquement de rencontrer certains membres de sa famille, voire l'entièreté de celle-ci. Dans ce cas, c'est la zizanie qui domine. Lorsque la rupture relationnelle est totale, Ego peut aussi s'abstenir de participer sans fournir d'alibi, en ne se souciant nullement d'offenser les membres de la parenté par son absence. Lorsqu'une famille est confrontée au refus catégorique de participation de certains de ses membres, en général, le rituel est dédoublé en plusieurs cellules.

En conclusion, avec la fête de Noël, laquelle véhicule un sens familial de nature intégrative, se rejouent annuellement l'emprise du noyau familial d'origine, la cohésion du groupe et la perméabilité des frontières internes à la famille. Au fil du temps, des ajustements ont lieu, mais dans un flou sépia permettant de donner une impression de tradition immuable à cette fête dont la forme rituelle est pourtant renégociée lors de changements familiaux (mariage, séparation, décès, naissance, etc.).

Evelyne Favart Docteur en sociologie Chargée de recherches FNRS, Université Libre de Bruxelles Avenue Jeanne 44 1050 Bruxelles, Belgique

\section{BIBLIOGRAPHIE}

Bawin-Legros, B. ; Gauthier, A. 1990. «Les grands-parents dans la dynamique familiale ", Relations intergénérationnelles. Parenté-transmission-mémoire, actes du colloque de Liège, 17-18 mai, p. 141-154. 
CAPlow, T. 1986. « Les cadeaux de Noël à Middletown ou comment faire respecter une règle sans pression apparente ", Dialogue, recherches cliniques et sociologiques sur le couple et la famille, p. 43-61, 91.

GODARD, F. 1992. La famille, affaire de générations, Paris, PUF.

Gotman, A. 1988. Hériter, Paris, Puf.

KaUfManN, J.C. 2002. "Secrets d'album », Un siècle de photos de famille, Paris, Ed. Textuel/Arte/Monum-Editions du Patrimoine.

Muxel, A. 1996. Individu et mémoire familiale, Paris, Nathan.

Perrot, M. 2000. Ethnologie de Noël, Paris, Grasset.

Segalen, M. 1998. Rites et rituels contemporains, Paris, Nathan.

Singly DE, F. 1993. Sociologie de la famille contemporaine, Paris, Nathan.

Singly DE, F. 1996. Le Soi, le couple, la famille, Paris, Nathan.

\section{Notes}

1. Le corpus est constitué, d'une part, d'entretiens sur le thème de Noël, réalisés au cours du second semestre 2002 par des étudiants, dans le cadre de travaux pratiques d'un enseignement de méthodologie qualitative, du département de sociologie de l'université de Liège. D'autre part, des entretiens réalisés dans le cadre de ma thèse de doctorat portant sur les liens entre frères et sœurs à l'âge adulte ont également été exploités.

2. Entretien réalisé par Céline Hazard.

3. Entretien réalisé par Véronique Cormann.
4. Entretien réalisé par Vincent Bichot.

5. Entretien réalisé par Belinda Herman.

6. Entretien réalisé par Bertrand Fincoeur.

7. Entretien réalisé par Emmanuelle Scheck.

8. Entretien réalisé par Céline Hazard.

9. Entretien réalisé par Olivia Guyaux.

10. Entretien réalisé par Hélène Seggio.

11. Entretien réalisé par Emmanuelle Scheck.

12. Entretien réalisé par Evelyne Favart.

13. Entretien réalisé par Evelyne Favart.

14. Entretien réalisé par Emmanuelle Scheck.

15. Voir à ce sujet les analyses fouillées de Gotman.

16. Entretien réalisé par Véronique Cormann.

17. Entretien réalisé par Emmanuelle Scheck.

18. Entretien réalisé par Evelyne Favart.

19. Ibid.

20. Ibid.

\section{RÉSUMÉ}

À partir d'une étude qualitative constituée d'entretiens compréhensifs, l'auteure, sociologue, étudie les enjeux familiaux autour de la fête de Noël, en examinant le sens que les personnes interviewées donnent à cette fête, la manière dont elles la vivent. Avec la fête de Noël, laquelle véhicule bien avant tout un sens familial de nature intégrative, se rejoue annuellement l'emprise du noyau familial d'origine.

\section{MoTS-CLÉS}

Sociologie, famille, intergénérationnel, rituel, Noël. 